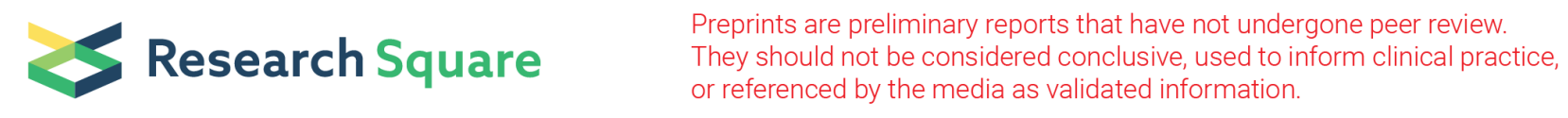

\title{
Sex Differences in Depressive Symptom Networks Among Community- Dwelling Older Adults in South Korea: A Network Comparison Test
}

\author{
Chiyoung Lee ( $\nabla$ clee33@uw.edu ) \\ University of Washington Bothell \\ Xiao Hu \\ Duke University
}

Research Article

Keywords: sex differences, depression, network analysis, older adults

Posted Date: November 2nd, 2021

DOI: https://doi.org/10.21203/rs.3.rs-1013910/v1

License: @ (i) This work is licensed under a Creative Commons Attribution 4.0 International License. Read Full License 


\section{Abstract}

This cross-sectional study investigated the sex differences in depressive symptom networks among community-dwelling older adults in Korea. The analysis was based on the 2019 Korean Community Health Survey data targeting older adults aged 65 years or older. Using network analysis, depressive symptom networks were constructed according to the items listed in the Patient Health Questionnaire- 9 for propensity score-matched male $(n=1,885)$ and female groups $(n=2,848)$. Strength centrality and network stability were tested. A network comparison test was performed to compare the global strength, network structure, and specific edge strength between the networks. Symptoms central to the network were similar between sexes, which were suicidal ideation, hopelessness, and psychomotor retardation/agitation. However, the global structure $(S=0.67, p=.008)$ and network structure $(M=0.11, p=.043)$ differed between sexes. The female symptom network showed more strengthened edges $\left(S_{\text {male }}=2.00 ; S_{\text {female }}=2.66\right)$. Particularly, four edges-loss of interest-hopelessness $(E=0.09, p=.016)$, sleep disturbance-low energy $/$ fatigue $(E=0.11, p=.005)$, loss of interestconcentration difficulty $(E=0.05, p=.047)$, and worthlessness-concentration difficulty $(E=0.08, p=.045)$-were more pronounced in the female network. Our results may help guide future research and clinical interventions for female depression.

\section{Introduction}

Depression is a global mental health concern that disproportionally affects females, with females being two times more likely to be diagnosed than males. ${ }^{1}$ Studies provide many explanations for this disparity, such as biological factors (e.g., endocrine), psychological factors (e.g., coping style), social factors (e.g., social roles and status, social support), or the possibility of the difference being artefactual (sex differences in symptom reporting, diagnostic bias). ${ }^{1}$ However, amid these explanations, another critical piece of evidence claims that the differences in the subtypes of depressive symptoms reported by males and females also play a role. ${ }^{2}$ For instance, studies consistently show that females are more likely than males to present with most subtypes of depression, including core symptoms of depression (e.g., depressed mood or diminished interest) and somatic symptoms (e.g., sleep disturbance or excessive fatigue) ${ }^{2}$ On the other hand, males tend to report more irritability, anger, risk-taking, and increased alcohol use, also known as the male depressive syndrome, which is not part of a major depressive diagnosis. ${ }^{3}$

However, many methodological issues can challenge traditional symptom-level analysis on exploring sex differences in depression prevalence. So far, these analyses have implicitly assumed that depressive symptoms arise from a common cause, analogous to classic physical disease models. ${ }^{4}$ In this model, symptoms are conceptualized as diagnostically equivalent and interchangeable ${ }^{5,6}$ and thus can be summed to produce an overall score indicating the severity of a mental disorder. ${ }^{7}$ In addition, different symptoms of mental disorder should co-occur when someone is experiencing the condition. These psychometric assumptions underlying the common cause model seem clinically implausible and obfuscates significant differences between symptoms on the one hand and between individuals on the other hand.

One recently proposed alternative is based on network models. Network models, which are based on network estimation and subsequent analysis of network topology, conceptualize a mental disorder as a syndromic constellation of symptoms that reciprocally interact and reinforce each other, and the network of symptoms itself is what constitutes the disorder. ${ }^{8}$ The network model assumes that the associations between symptoms of depression may be different for each sex, and thus, might help to explain sex differences in depression prevalence rates. ${ }^{9}$ Importantly, network analysis incorporates sophisticated techniques to study the complex patterns of symptom interactions. For instance, using network analysis, influential symptoms (i.e., those connected with most symptoms or with stronger connections) can be detected, which might be important factors for understanding sex differences in depression. Finally, some properties (e.g., network structure, overall connectivity) of networks can be compared between groups of individuals. Until now, only a few studies evaluated sex differences in a network structure of depressive symptoms, either among adolescents or broad-aged populations (e.g., between $18-70$ years). ${ }^{10-12}$

Depression is the most common mental disorder and a leading cause of disability among older adults. ${ }^{13}$ With the drastic increase of an aging population in South Korea (hereafter "Korea") and many other countries, there is also a much higher prevalence of depression in the older age group than in other age groups. ${ }^{14}$ In particular, an increased prevalence of depression in older Korean females than older males has been reported consistently over time. ${ }^{15}$ Perhaps there may be differences in the depressive symptom networks between sexes that may help explain this increased vulnerability for females. However, to our knowledge, no network analysis has examined how the network of depressive symptoms differs across sexes among the older adult population.

\section{Specific aim of the study}

This study is an explorative assessment of sex differences in depressive symptom networks among community-dwelling older adults in Korea. Specifically, we compared the symptom networks of both sexes using network function and evaluated which symptoms and associations between symptoms are most influential in each network. Next, we performed a network comparison test to compare the specific properties of male and female symptom networks. Finally, even if the networks of different sexes differed, the cause for such differences can still be unclear as other covariates may have affected the severity of depression. For this reason, we matched participants from each different sex group using propensity score matching (PSM) and created a comparable group.

\section{Methods}




\section{Study design}

This is a cross-sectional study utilizing the 2019 Korean Community Health Survey (KCHS) data. The KCHS is a nationwide health interview survey carried out by the Korean Centers for Disease Control and Prevention (KCDC) to estimate the pattern of disease prevalence and morbidity, as well as to understand the personal lifestyle and health behavior, of adults aged 19 years or older. The KCHS comprises free public data and does not include personal information. All participants provided informed consent and all study protocols and procedures of the KCHS were reviewed and approved by the KCDC institutional review board. Detailed descriptions of the KCHS are on their website (https://chs.cdc.go.kr/chs/main.do).

In addition, the institutional review board of University of Washington approved this study as an exempt study (protocol number: STUDY00014343). All methods were performed in accordance with the relevant guidelines and regulations.

\section{Study sample}

The study population was older adults aged 65 years or older. There was a total of 74,497 people aged 65 years or older (56.2\% female) who participated in the KCHS survey in 2019. First, from this entire group, we excluded participants categorized as having no or minimal depression (those with a score of 0-4 in the total sum of the nine-item Patient Health Questionnaire [PHQ-9] score) and only selected people with clinical depression with complete data $(n=14,262)$. We also excluded participants who reported experiencing disruptions to daily functioning due to cognitive impairments during the past year, as these disruptions may complicate the assessment of depression; this resulted in 7,166 participants. Finally, from this, we excluded data with any missing values within variables of interest (e.g., covariates for PSM analysis). This process generated a total of 7,023 (70.0\% female) baseline samples.

\section{Measurement}

Depressive symptoms were assessed using the PHQ-9 using nine symptoms that make up the diagnosis of major depressive disorders, rating each item on a 0-3 scale based on whether the symptom bothered the respondent in the last two weeks (Table 1). Scores included 0: not at all, 1: several days, 2: more than half the days, or 3: nearly every day. The items are summed for a total score. A cut-off point of 0-4 indicates no to minimal depressive symptoms, 5-9 mild depressive symptoms, 10-14 moderate depressive symptoms, 15-19 moderately severe depressive symptoms, and 20-27 severe depressive symptoms. The PHQ-9 is a well-established validated tool with high accuracy for screening, recommended in clinical practice. $^{16}$

Table 1

PHQ-9 items

\begin{tabular}{|c|c|c|c|}
\hline Item & $\begin{array}{l}\text { Labels in } \\
\text { the figure }\end{array}$ & Symptom & Questionnaire ${ }^{a}$ \\
\hline $\begin{array}{l}\text { Item } \\
\# 1\end{array}$ & Lost_int & Loss of interest & Little interest or pleasure in doing things \\
\hline $\begin{array}{l}\text { Item } \\
\# 2\end{array}$ & Hope & $\begin{array}{l}\text { Feelings of } \\
\text { hopelessness }\end{array}$ & Feeling down, depressed, or hopeless \\
\hline $\begin{array}{l}\text { Item } \\
\text { \#3 }\end{array}$ & $S / p$ & Sleep disturbance & Trouble falling or staying asleep, or sleeping too much \\
\hline $\begin{array}{l}\text { Item } \\
\# 4\end{array}$ & Ener & $\begin{array}{l}\text { Low energy or } \\
\text { fatigue }\end{array}$ & Feeling tired or having little energy \\
\hline $\begin{array}{l}\text { Item } \\
\# 5\end{array}$ & App & Appetite problems & Poor appetite or overeating \\
\hline $\begin{array}{l}\text { Item } \\
\# 6\end{array}$ & Worth & $\begin{array}{l}\text { Feelings of } \\
\text { worthlessness }\end{array}$ & Feeling bad about yourself or that you are a failure or have let yourself or your family down \\
\hline $\begin{array}{l}\text { Item } \\
\# 7\end{array}$ & Conc & $\begin{array}{l}\text { Concentration } \\
\text { difficulty }\end{array}$ & Trouble concentrating on things, such as reading the newspaper or watching television \\
\hline $\begin{array}{l}\text { Item } \\
\text { \#8 }\end{array}$ & Motor & $\begin{array}{l}\text { Psychomotor } \\
\text { retardation or } \\
\text { agitation }\end{array}$ & $\begin{array}{l}\text { Moving or speaking so slowly that other people could have noticed. Or the opposite being so fidgety } \\
\text { or restless that you have been moving around a lot more than usual }\end{array}$ \\
\hline $\begin{array}{l}\text { Item } \\
\# 9\end{array}$ & Suic & Suicidal ideation & Thoughts that you would be better off dead, or of hurting yourself \\
\hline
\end{tabular}

\section{Covariates for propensity score matching}

Covariates were selected based on data availability and previous depression research on older adults. The covariates included: age (continuous), residential location (urban or rural), living arrangement (living alone, living with a spouse, or living with family or others), income (< 1 million KRW, 1-2 million KRW, 2-3 million KRW, or $\geq 3$ million KRW), current employment status (yes or no), education (no formal education, elementary school, middle 
school, or high school or higher), social participation (yes or no), physical activity (yes or no), and chronic conditions (yes or no). Residential location was classified as "urban" if the participant lived in a dong (neighborhood) in the administrative division or as "rural" if the respondent lived in an eup (town) or a myeon (township). Regarding social participation, the answer is yes when older adults participate in one of the following activities: religious activity, friendship activity, leisure activity, or charity activity. The chronic condition includes having hypertension and/or diabetes.

\section{Propensity score matching}

Among total participants, male participants were statistically matched (1:1.5) with female participants according to PSM without replacement (male $\mathrm{n}$ $=1,885$; female $n=2,848$ ). Logistic regression was used to generate propensity scores. Matching was performed using the nearest neighbor algorithm with a caliper distance of 0.25 . A caliper of 0.25 standard deviations of the propensity score was chosen, as this has been recommended as a reasonable distance for reducing bias between groups. ${ }^{17}$ This procedure was conducted by R-package Match/t.

\section{Statistical analysis}

The balance of the covariates and PHQ-9 scores was assessed before and after PSM using $\chi^{2}$ tests and $t$-tests for continuous variables. Next, depressive symptom networks were constructed for propensity-score matched male and female groups according to the items listed in the $\mathrm{PHQ}-9$ using the network analysis. Especially, we structured our findings using the four steps identified by Fried et al. ${ }^{18}$ : (a) network estimation, (b) network characterization, (c) network stability, and (d) network comparison. All network analyses were carried out using R version 3.6.2. All other statistical analyses were conducted with SAS software.

\section{(a) Network estimation}

Following the recommendations for network analysis with cross-sectional ordinal data by Epskamp and colleagues, ${ }^{19}$ R-package $q g r a p h$ was used to create partial correlation networks for both sex groups. To estimate our network models, we chose the "Graphical Least Absolute Shrinkage and Selection Operator (LASSO) with extended Bayesian Information Criterion (EBIC)" method of the package. When LASSO is used to estimate a network, it avoids overfitting by shrinking the partial correlation coefficients, so small coefficients are estimated to be exactly zero, indicating conditional independence and making the interpretability of the network structure easier. ${ }^{19}$ The EBIC encompasses a hyperparameter $\mathrm{Y}$ (gamma) that determines to what degree simpler models (fewer edges) should be preferred by the EBIC. In this study, we set the value of $y$ to the default value of 0.25 . In the resulting network (a Gaussian Graphical Model), nodes represent symptoms of depression, and edges represent partial correlations between symptoms.

\section{(b) Network characterization}

To assess and quantify the importance of each node in the present network, we computed three major centrality indices: strength (i.e., the sum of all the edge weights directed to a single node), betweenness (i.e., number of times that node lies on the shortest path between two other nodes), and closeness (i.e., the average distance from a node to all other ones) ${ }^{20}$ Generally, nodes with higher centrality are more central to the network and have more frequent and stronger relationships with other nodes than nodes with lower centrality. Of note, research has indicated that strength centrality is crucial for psychological networks as it reflects the likelihood that activation of a certain symptom will be followed by the activation of other symptoms. $^{21}$

\section{(c) Network stability}

We examined the stability of the network by using R-package bootnet. First, the accuracy of edge weights was evaluated by calculating $95 \%$ confidence intervals using a non-parametric bootstrap approach (1,500 bootstrap samples). Second, the stability of node strengths was evaluated by computing the correlation stability (CS) coefficient using a case-dropping bootstrap approach. Epskamp et al. ${ }^{22}$ suggest only interpreting centrality indices with a CS-coefficient above 0.25 but preferentially above 0.5 , with 0.7 as the benchmark. Lastly, bootstrapped difference tests ( 1,500 bootstrap samples and $a=0.05$ ) for edge weights and node strengths were conducted to evaluate whether two edge weights or two node strengths differed significantly from one another.

\section{(d) Network comparison}

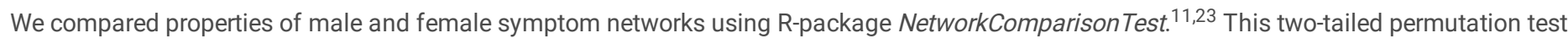
enables calculating the difference between two networks via repeated and random regrouping of individuals. In particular, this test investigates differences in network invariance (i.e., equality in network properties) at three levels: global strength (assuming that overall connectivity in both networks is exactly the same), network structure (assuming that the structure of both networks is exactly the same), and one-to-one edge strength (assuming that each specific edge is identical across cohorts). Edge strength invariance was only tested when a lack of network structure invariance is upheld.

\section{Results}

The results were "unweighted" as we could not incorporate a complex survey design (e.g., sampling weights, strata, and primary sampling units) into analyses due to the current lack of network methodologies for complex design features. Hereafter, we refer to unweighted prevalence rates (or 
unweighted means) directly as prevalence rates (or means) and provide further discussion in the limitation section.

According to Table 2, before PSM, significant differences were detected between male and female groups according to their age, living arrangements, income, current employment status, education, social participation, and chronic conditions. After PSM, differences were observed concerning living arrangements, income, current employment status, and education (however, the $p$-values for these comparisons overall increased). Table 2 also shows that the sum-score of PHQ-9 was constantly significantly higher in the female group than in the male group regardless of the matching procedure ( $p<$ .05). However, the $p$-value changed from <.001 to .039 after PSM. For individual symptoms, before PSM, females had significantly greater symptom severity compared to males for five symptoms: Hope, SIp, Ener, App, Suic. After PSM, this pattern hardly changed except for Suic.

Table 2. General differences before and after PSM 


\begin{tabular}{|c|c|c|c|c|c|c|}
\hline \multirow[t]{4}{*}{ Variable } & \multicolumn{3}{|l|}{ Before PSM } & \multicolumn{3}{|l|}{ After PSM } \\
\hline & \multicolumn{2}{|c|}{ Mean \pm SD or $\%$} & \multirow[t]{3}{*}{$p$-value } & \multicolumn{2}{|c|}{ Mean \pm SD or $\%$} & \multirow[t]{3}{*}{$p$-value } \\
\hline & Male & Female & & Male & Female & \\
\hline & $(\mathrm{n}=2,109)$ & $(n=4,914)$ & & $(n=1,885)$ & $(n=2,848)$ & \\
\hline \multicolumn{7}{|l|}{ Covariates } \\
\hline Age & $75.12 \pm 6.57$ & $75.79 \pm 6.72$ & $<.001$ & $74.89 \pm 6.54$ & $74.81 \pm 6.72$ & .674 \\
\hline Residential location & & & .466 & & & .310 \\
\hline Rural & 57.3 & 58.3 & & 56.7 & 55.2 & \\
\hline Urban & 42.7 & 41.7 & & 43.3 & 44.8 & \\
\hline Living arrangement & & & $<.001$ & & & $<.001$ \\
\hline Living alone & 18.4 & 39.3 & & 20.3 & 25.4 & \\
\hline Living with a spouse & 62.2 & 34.7 & & 58.6 & 50.8 & \\
\hline Living with a family & 19.4 & 26.0 & & 21.1 & 23.8 & \\
\hline Income & & & $<.001$ & & & .036 \\
\hline$<1$ million KRW & 39.4 & 52.2 & & 39.9 & 44.2 & \\
\hline 1-2 million KRW & 30.2 & 22.8 & & 29.7 & 27.3 & \\
\hline 2-3 million KRW & 15.0 & 10.6 & & 14.1 & 13.1 & \\
\hline$\geq 3$ million KRW & 15.5 & 14.4 & & 16.2 & 15.4 & \\
\hline Current employment status & & & $<.001$ & & & .017 \\
\hline No & 63.0 & 73.2 & & 65.1 & 68.4 & \\
\hline Yes & 37.0 & 26.8 & & 34.9 & 31.6 & \\
\hline Education & & & $<.001$ & & & $<.001$ \\
\hline No formal education & 17.3 & 47.8 & & 19.3 & 25.9 & \\
\hline Elementary school & 35.5 & 33.3 & & 38.4 & 42.3 & \\
\hline Middle school & 20.7 & 10.5 & & 19.5 & 17.2 & \\
\hline High school or higher & 26.6 & 8.5 & & 22.8 & 14.5 & \\
\hline Social participation & & & $<.001$ & & & .567 \\
\hline No & 45.3 & 40.5 & & 43.9 & 43.1 & \\
\hline Yes & 54.7 & 59.5 & & 56.1 & 56.9 & \\
\hline Physical activity & & & 0.614 & & & .827 \\
\hline No & 65.9 & 65.3 & & 65.1 & 64.8 & \\
\hline Yes & 34.1 & 34.7 & & 34.9 & 35.2 & \\
\hline Chronic conditions & & & $<.001$ & & & .060 \\
\hline No & 41.0 & 32.4 & & 40.0 & 37.3 & \\
\hline Yes & 59.0 & 67.6 & & 60.0 & 62.7 & \\
\hline \multicolumn{7}{|l|}{ PHQ-9 } \\
\hline Sum score & $7.44 \pm 3.26$ & $7.78 \pm 3.52$ & $<.001$ & $7.49 \pm 3.31$ & $7.70 \pm 3.48$ & .039 \\
\hline Lost_int & $1.04 \pm 1.00$ & $0.99 \pm 0.95$ & .088 & $1.05 \pm 1.00$ & $1.02 \pm 0.96$ & .291 \\
\hline Hope & $0.92 \pm 0.95$ & $0.98 \pm 0.85$ & .003 & $0.93 \pm 0.85$ & $0.99 \pm 0.85$ & .008 \\
\hline$S / p$ & $1.67 \pm 1.12$ & $1.82 \pm 1.08$ & $<.001$ & $1.69 \pm 1.11$ & $1.79 \pm 1.08$ & .004 \\
\hline Ener & $1.50 \pm 0.95$ & $1.57 \pm 0.92$ & $<.001$ & $1.50 \pm 0.96$ & $1.58 \pm 0.92$ & .004 \\
\hline$A p p$ & $0.90 \pm 0.94$ & $1.03 \pm 0.99$ & $<.001$ & $0.89 \pm 0.93$ & $1.04 \pm 0.97$ & $<.001$ \\
\hline
\end{tabular}




\begin{tabular}{|lllllll|}
\hline Worth & $0.40 \pm 0.68$ & $0.36 \pm 0.66$ & .004 & $0.41 \pm 0.68$ & $0.36 \pm 0.66$ & .010 \\
\hline Conc & $0.38 \pm 0.71$ & $0.36 \pm 0.70$ & .360 & $0.39 \pm 0.71$ & $0.34 \pm 0.68$ & .033 \\
\hline Motor & $0.27 \pm 0.62$ & $0.25 \pm 0.61$ & .075 & $0.28 \pm 0.63$ & $0.23 \pm 0.60$ & .020 \\
\hline Suic & $0.37 \pm 0.67$ & $0.42 \pm 0.72$ & .002 & $0.37 \pm 0.67$ & $0.39 \pm 0.68$ & .307 \\
\hline
\end{tabular}

Note: SD = Standard Deviation

Figure 1 shows a separate network consisting of nine symptoms constructed for males and females. In this network, blue edges represent positive relationships, and a red edge denotes a negative relationship between symptoms. The thickness and saturation of the edges signify their magnitude. Grossly, the female symptom network showed more strengthened edges. The overall network connection was sparse in the male network. In the male network, the four greatest edge weights were between Worth and Suic (part r = 0.42), Lost_int and Hope (part $r=0.34)$, Motor and Suic (part $r=0.32$ ), and Conc and Motor (part $r=0.31$ ). In the female network, the strongest edges were between Lost_int and Hope (part $r=0.43)$, Worth and Suic (part $r=$ 0.41), and Conc and Motor (part $r=0.40$ ).

In both networks, node strength showed better stability than closeness and betweenness; strength [CS (cor $=0.7)=0.75$ for both male and female network], closeness [CS (cor $=0.7)=0.67$ for male, 0.59 for female network], and betweenness [CS (cor $=0.7)=0.13$ for male and 0 for female network]. Given that only the CS-coefficient for node strength reached the benchmark value of 0.7 and the network seems to be more stable in that parameter of centrality, we only interpreted strength centrality (see Supplementary Fig. S3 for more details).

Figure 2 displays the centrality measures in both symptom networks. In the male network, inspection of the node strength centrality plot indicated that Suic had the greatest node strength. Worth and Motor also had particular salience and influence in the network. In particular, Suic (strength $=1.56$ ), Worth (strength $=1.00$ ), and Motor (strength $=0.77$ ) had significantly greater strength than $75.0 \%, 62.5 \%$, and $62.5 \%$ of the other nodes in the network, respectively. In the female network, Suic showed the highest node strength. Motor and Worth were also highly influential nodes in the network. Especially, Suic (strength $=1.18$ ), Motor (strength $=1.00$ ), and Worth (strength $=0.95$ ) had significantly higher strength than $62.5 \%, 75.0 \%$, and $62.5 \%$ of the other nodes, respectively (see Supplementary Fig. S4 for bootstrapped significance test results). On the other hand, SIp, Ener, and App had the lowest node strengths.

The network comparison test revealed that both networks were invariant in terms of global strength: $\mathrm{S}\left(\mathrm{G}_{\text {male, }}, \mathrm{G}_{\mathrm{female}}\right)=0.67, p=.008$ (global strength by sex: $\left.\mathrm{S}_{\text {male }}=2.00 ; \mathrm{S}_{\text {female }}=2.66\right)$. In addition, the network structure invariance test was significant: $M\left(G_{\text {male }}, G_{\text {female }}\right)=0.11, p=.043$. Given this, differences between specific edges were tested. The invariant edge strength test revealed significantly different correlations between samples in: Lost_int-Hope, $\mathrm{E}_{\mathrm{LH}}\left(\mathrm{G}_{\text {male }}, \mathrm{G}_{\text {female }}\right)=0.09, p=.016 ;$ Slp-Ener, $\mathrm{E}_{\mathrm{SE}}\left(\mathrm{G}_{\text {male, }} \mathrm{G}_{\text {female }}\right)=0.11, p=.005 ;$ Lost_int-Conc, $\mathrm{E}_{\mathrm{LC}}\left(\mathrm{G}_{\mathrm{male}}, \mathrm{G}_{\mathrm{female}}\right)=0.05, p=.047$; and Worth-Conc, $\mathrm{E}_{\mathrm{WC}}\left(\mathrm{G}_{\text {male, }} \mathrm{G}_{\text {female }}\right)=0.08, p=.045$. In this sense, these two correlations were significant in the female symptom network, but shrunk from the male symptom network, due to their low magnitude. The results of the network structure and global strength invariance test are also plotted in Figure 3. Figure 3 shows the reference distributions-created by permutations of the data-with which the test statistics $S$ (i.e., the difference in global strength) and $\mathrm{M}$ (i.e., the maximum difference in edge strength of the two networks) can be evaluated.

\section{Discussion}

While individual symptom endorsement and the overall summed scores differed, depressive symptoms exhibiting the greatest importance within the networks were highly similar between sexes: suicidal ideation, worthlessness, and psychomotor retardation/agitation had the highest values of strength centrality in both male and female networks. Moreover, the edge connecting suicidal ideation and worthlessness was identified as one of the central edges in both networks. This aligns with Murri et al.'s study, ${ }^{24}$ which identified death wishes and pessimism as most central in the network of late-life depressive symptoms for both sexes (mean age $=74$ years, $59 \%$ female). Our results also support the meta-analytic findings of Cavanagh et al., ${ }^{2}$ who identified no sex differences in depressive symptoms with regard to suicidality or psychomotor retardation. Indeed, evidence supports that older adults with depression often present with pathogenic reactions (e.g., suicidal ideation, feelings of worthlessness) instead of the normal intense

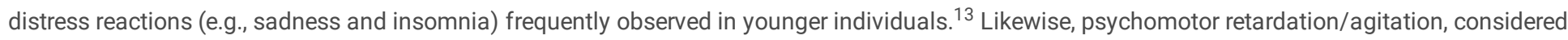
as a variant of primary degenerative dementia, is common in both major and minor late-life depression. ${ }^{25}$ Therefore, when examining the network of late-life depressive symptoms, it makes sense that we find this unique symptom profile as the central hub, regardless of sex.

However, we found significant sex differences in measures of global strength and network structure. Although not directly comparable, this finding seems to contrast with results of previous research that has examined cross-sex differences in network structures of depressive symptoms in individuals seeking treatment for major depressive disorder. ${ }^{9,12}$ Van Borkulo et al. ${ }^{11}$ also conducted a similar study with male and female adults who had experienced a depressive episode and found no sex differences in global strength. Notably, in the current study, older female patients had denser or more tightly connected symptom networks, as global strength was higher in the female group than in the male group. According to the network perspective, a more densely and strongly connected network indicates that an individual feels more "trapped" in the disordered state (i.e., depression) than someone with a less densely connected network. ${ }^{26}$ Particularly, more strongly connected networks will feature stronger feedback among their symptoms and thus may be related to greater vulnerability to depression and a less positive prognosis. ${ }^{26}$ In contrast, we found that older male 
patients had less dense symptom networks or weaker connections between symptoms. Therefore, they might be more resistant to symptom spreading and have protection against developing depression.

Specifically, one-to-one edge strength tests showed that the magnitudes of the following four edges in the female symptom network were significantly stronger than those in the male symptom network: loss of interest-hopelessness, sleep disturbance-low energy/fatigue, loss of interestconcentration difficulty, and worthlessness-concentration difficulty. Associations among these symptoms may help explain the sex disparity in depression rates among older adults. Although typical "female depression" with a specific symptom profile has not been identified in older adults, it is worth noting that these symptoms characterize the "depletion syndrome" more commonly found specifically among older female adults. ${ }^{27}$ The depletion syndrome's form differs somewhat across modeling approaches, but its stable features include deficits in self-worth, no interest in pleasurable activities, decreased energy, a general sense of hopelessness, and psychomotor retardation. ${ }^{28,29}$ In fact, the finding that psychomotor retardation/agitation exerted a unique influence in the female network indirectly supports this argument.

Among the four edges, the edge between loss of interest and hopelessness, which was also identified as one of the central edges of the female symptom network, seems especially important and novel. Suh et al. ${ }^{30}$ also found this association in their network analysis study with a community sample of Korean adults (age 20-86 years, 51.5\% female). Importantly, these two symptoms represent core elements of suicidal ideation in older adults and are key predictors of suicide, equal in power if not more powerful compared to depression alone. ${ }^{31}$ Thus, these symptoms should be as thoroughly assessed as other core features of late-life depression among older female adults. The finding that the edge between sleep disturbance and low energy/fatigue was more pronounced in female symptom networks than in male symptom networks accords with previous research. ${ }^{2,11}$ However, in this study, sleep disturbance and low energy/fatigue were identified as peripheral symptoms in the female symptom network based on the centrality test, even though empirical data suggest that their prevalence is high. ${ }^{32}$ A potential explanation is that such somatic symptoms are prevalent in late life regardless of whether they are caused by depression, medical illnesses, or an interaction of the two. ${ }^{33}$

The other edges-loss of interest-concentration difficulty and worthlessness-concentration difficulty-appear appropriate for substantive interpretation. It is well established that cognitive functions such as concentration and attention are distorted in late-life depression, and they possess adequate clinical utility for diagnosing depression in older adults. ${ }^{13}$ However, to our knowledge, previous geriatric studies have not found associations of concentration difficulties with other symptoms such as loss of interest and worthlessness. Accordingly, this finding may inform theoretical considerations about sex differences in depression among older adults. Simultaneously, future studies should examine treatments aiming to deactivate these symptoms. Although not perfectly analogous, $\mathrm{Kim}^{34}$ showed that a program combining physical and recreational activities such as arts and crafts considerably improved cognitive function (self-esteem, creativity, motor function, concentration, memory) and decreased depression levels among older female adults in Korea.

Nevertheless, the present findings should be interpreted with caution. After the PSM, sex differences in participants' characteristics persisted, especially with respect to living arrangements and education. These factors may account for differences in the symptom networks observed in the present study. For instance, there were more individuals living alone in the female group compared to the male group, which may explain the increased negative cognitive features presenting in the female network. ${ }^{35}$ Further, female participants tended to have lower levels of education. Education is correlated with the capacity to direct attention, especially among older women ${ }^{36}$; this may support the results regarding the concentration difficulty node's higher connection in the symptom network compared to other symptoms.

This study has several limitations. First, we could not incorporate complex design features of KCHS due to the lack of established methods for network models. Accordingly, this sample should not be considered truly representative of community-dwelling Korean older adults in 2019. Instead, researchers must view our results as those emerging from the analysis of an extensive national survey with a diverse group of participants and with generalizability possibly superior to smaller/convenience samples. In the same vein, it should also be noted that the NCT has only recently been proposed as a method for comparing network structures between groups. Thus, such methods should be interpreted with some caution as they gain more support and as other methods of network comparisons emerge. Second, a self-reported method for assessing depressive symptoms among older adults can lead to misinterpretation and biased responses, which may lower the precision of the analyses. Third, associations between symptoms within the network do not infer causal relationships as the current study used cross-sectional data; although a central symptom is likely to have an influence on other nodes, it may be a more efficient target for intervention if associations with other symptoms are directed outward or at least bidirectional. Fourth, we excluded many female subjects during the PSM, which is a primary shortcoming of the PSM methodology. Fifth, while it is not uncommon for depression scales to have minimal content overlap, having different types of content in scales might generate a different psychopathology network. Accordingly, one should be careful when comparing this study's results with others' results. Another potential limitation of these analyses is the relatively low mean level of depression symptoms (male $=7.49 \pm 3.31$, female $=7.70 \pm 3.48$ after PSM). There is some preliminary evidence that symptom network connectivity, though not necessarily structure, differs according to levels of symptom severity. ${ }^{37}$ Future studies should examine whether the depressive symptom network structure varies across different levels of depression severity and among patients meeting criteria for depressive syndromes.

\section{Conclusion}


This network analysis of depressive symptoms in community-dwelling older adults in Korea revealed that the two independently estimated male and female networks had highly similar central symptoms. However, significant sex differences in measures of global strength and network structure emerged. Older female adults tend to have denser or more strongly connected symptom networks; therefore, symptoms may develop more quickly or strongly for them compared to men. This may help explain the increased vulnerability to depression among older women. Particularly, four edges were significantly stronger in the female symptom network, namely loss of interest-hopelessness, sleep disturbance-low energy/fatigue, loss of interestconcentration difficulty, and worthlessness-concentration difficulty. These might be crucial factors for understanding the sex disparity in depression rates. The present study's findings may help guide future research and clinical interventions for female depression. It is also important to educate health professionals on the differences in depressive symptom presentation to ensure appropriate treatment for older female adults.

\section{Declarations}

\section{Acknowledgements}

None.

\section{Author contributions}

L.C. contributed to the design and implementation of the research, to the analysis of the results and to the writing of the manuscript. H.X. provided critical feedback, helped shape the research, analysis and manuscript, and approved the final submitted version.

\section{Competing interest}

The authors declare no competing interests.

\section{Funding}

There was no funding for the secondary analysis of the public data.

\section{References}

1. Eid, R. S., Gobinath, A. R., \& Galea, L. A. Sex differences in depression: Insights from clinical and preclinical studies. Prog. Neurobiol. 176, 86-102 (2019).

2. Cavanagh, A., Wilson, C. J., Kavanagh, D. J., \& Caputi, P. Differences in the expression of symptoms in men versus women with depression: a systematic review and meta-analysis. Harv. Rev. Psychiatry. 25, 29-38 (2017).

3. Azorin, J. M. et al. Gender differences in a cohort of major depressive patients: further evidence for the male depression syndrome hypothesis. J. Affect. Disord. 167, 85-92 (2014).

4. Insel, T. R. The NIMH research domain criteria (RDoC) project: precision medicine for psychiatry. Am. J. Psychiatry. 171, 395-397 (2014).

5. Cramer, A. O., Waldorp, L. J., Van Der Maas, H. L., \& Borsboom, D. Comorbidity: A network perspective. Behav. Brain. Sci. 33, 137-150 (2010).

6. Lux, V., \& Kendler, K. S. Deconstructing major depression: a validation study of the DSM-IV symptomatic criteria. Psychol. Med. 40, 1679-1690 (2010).

7. Fried, E. I., \& Nesse, R. M. Depression sum-scores don't add up: why analyzing specific depression symptoms is essential. BMC Med. 13, 1-11 (2015).

8. Borsboom, D., \& Cramer, A. O. Network analysis: an integrative approach to the structure of psychopathology. Annu. Rev. Clin. Psychol. 9, 91-121 (2013).

9. Ginger, E. J. Sex Differences in a Network Model of Depressive Symptoms. (Illinois Institute of Technology, 2021).

10. Mullarkey, M. C., Marchetti, I., \& Beevers, C. G. Using network analysis to identify central symptoms of adolescent depression. J. Clin. Child Psychol. 48, 656-668 (2019).

11. Van Borkulo, C. et al. Comparing network structures on three aspects: A permutation test. https://www.researchgate.net/profile/Claudia-VanBorkulo-

2/publication/314750838_Comparing_network_structures_on_three_aspects_A_permutation_test/links/5ff5ac0345851553a022c099/Comparingnetwork-structures-on-three-aspects-A-permutation-test.pdf (2017).

12. Vetter, J. S. et al. Sex differences in depressive symptoms and their networks in a treatment-seeking population-a cross-sectional study. J. Affect. Disord. 278, 357-364 (2021).

13. Sözeri-Varma, G. Depression in the elderly: clinical features and risk factors. Aging Dis. 3, 465 (2012).

14. Park, J. H. et al. A nationwide survey on the prevalence and risk factors of late life depression in South Korea. J. Affect. Disord. 138, 34-40 (2012).

15. Park, S. Y. The effects of health-related factors and social networks on depressive symptoms in elderly men and women: focusing on the moderating effects of gender. Health. Soc. Care. Community. 38, 154-190 (2018). 
16. Zimmerman, M. Using the 9-Item Patient Health Questionnaire to screen for and monitor depression. Jama. 322, 2125-2126 (2019).

17. Stuart, E. A. Matching methods for causal inference: A review and a look forward. Stat Sci. 25, 1-21 (2010).

18. Fried, E. I. Replicability and generalizability of PTSD networks: A cross-cultural multisite study of PTSD symptoms in four trauma patient samples. Clin. Psychol. Sci. 10.17605/OSF.IO/3ZQ5U (2017).

19. Epskamp, S., Kruis, J., \& Marsman, M. Estimating psychopathological networks: Be careful what you wish for. PloS One. 12, e0179891; 10.1371/journal.pone.0179891 (2017).

20. Valente, T. W. Network interventions. Science. 337, 49-53 (2012).

21. Bringmann, L. F. et al. What do centrality measures measure in psychological networks? J. Abnorm. Psychol. 10.1037/abn0000446 (2019).

22. Epskamp, S., Borsboom, D., \& Fried, E. I. Estimating psychological networks and their accuracy: A tutorial paper. Behav. Res. Methods. 50, 195-212 (2018).

23. Van Borkulo, C., Epskamp, S., Jones, P., Haslbeck, J., \& Millner, A. Package 'NetworkComparisonTest'. https://cran.microsoft.com/web/packages/NetworkComparisonTest/NetworkComparisonTest.pdf (2016).

24. Murri, M. B., Amore, M., Respino, M., \& Alexopoulos, G. S. The symptom network structure of depressive symptoms in late-life: Results from a European population study. Mol. Psychiatry. 25, 1447-1456 (2020).

25. Story, T. J., Potter, G. G., Attix, D. K., Welsh-Bohmer, K. A., \& Steffens, D. C. Neurocognitive correlates of response to treatment in late-life depression. Am J Geriatr Psychiatry. 16, 752-759 (2008).

26. Van Borkulo, C. et al. Association of symptom network structure with the course of depression. JAMA Psychiatry. 72, 1219-1226 (2015).

27. Newmann, J. P., Engel, R. J., \& Jensen, J. E. Age differences in depressive symptom experiences. J. Gerontol. 46, P224-P235 (1991).

28. Fernandez-Guasti, A., Fiedler, J. L., Herrera, L., \& Handa, R. Sex, stress, and mood disorders: at the intersection of adrenal and gonadal hormones. Horm. Metab. Res. 44, 607-618 (2012).

29. Gatz, M., \& Fiske, A. Aging women and depression. Prof. Psychol.: Res. Pract. 34, 3-9 (2003).

30. Suh, W. Y., Lee, J., Yun, J. Y., Sim, J. A., \& Yun, Y. H. A network analysis of suicidal ideation, depressive symptoms, and subjective well-being in a community population. J. Psychiatr. Res. 142, 263-271 (2021).

31. Hendricks, P. S., \& Grob, C. S. Classic Psychedelics and Rational Suicide in the Elderly. in Rational suicide in the Elderly: Exploring the Potential Utility of a Reemerging Treatment Paradigm. 203-210 (Springer Cham, 2017).

32. Hegeman, J. M., Kok, R. M., Van der Mast, R. C., \& Giltay, E. J. Phenomenology of depression in older compared with younger adults: metaanalysis. Br J Psychiatry. 200, 275-281 (2012).

33. Haigh, E. A., Bogucki, O. E., Sigmon, S. T., \& Blazer, D. G. Depression among older adults: a 20-year update on five common myths and misconceptions. Am J Geriatr Psychiatry. 26, 107-122 (2018).

34. Kim, D. The effects of a combined physical activity, recreation, and art and craft program on ADL, cognition, and depression in the elderly. J. Phys. Ther. Sci. 29, 744-747 (2017).

35. Lee, S. H. et al. Influence of loneliness on cognitive decline among elderly living alone in Korea: one year prospective study. $J$ Korean Acad Fam Med. 29, 695-702 (2008).

36. Jansen, D. A., \& Keller, M. L. Cognitive function in community-dwelling elderly women: attentional demands and capacity to direct attention. J. Gerontol. Nurs. 29, 34-43 (2003).

37. Santos Jr, H., Fried, E. I., Asafu-Adjei, J., \& Ruiz, R. J. Network structure of perinatal depressive symptoms in Latinas: relationship to stress and reproductive biomarkers. Res Nurs Health. 40, 218-228 (2017).

\section{Figures}




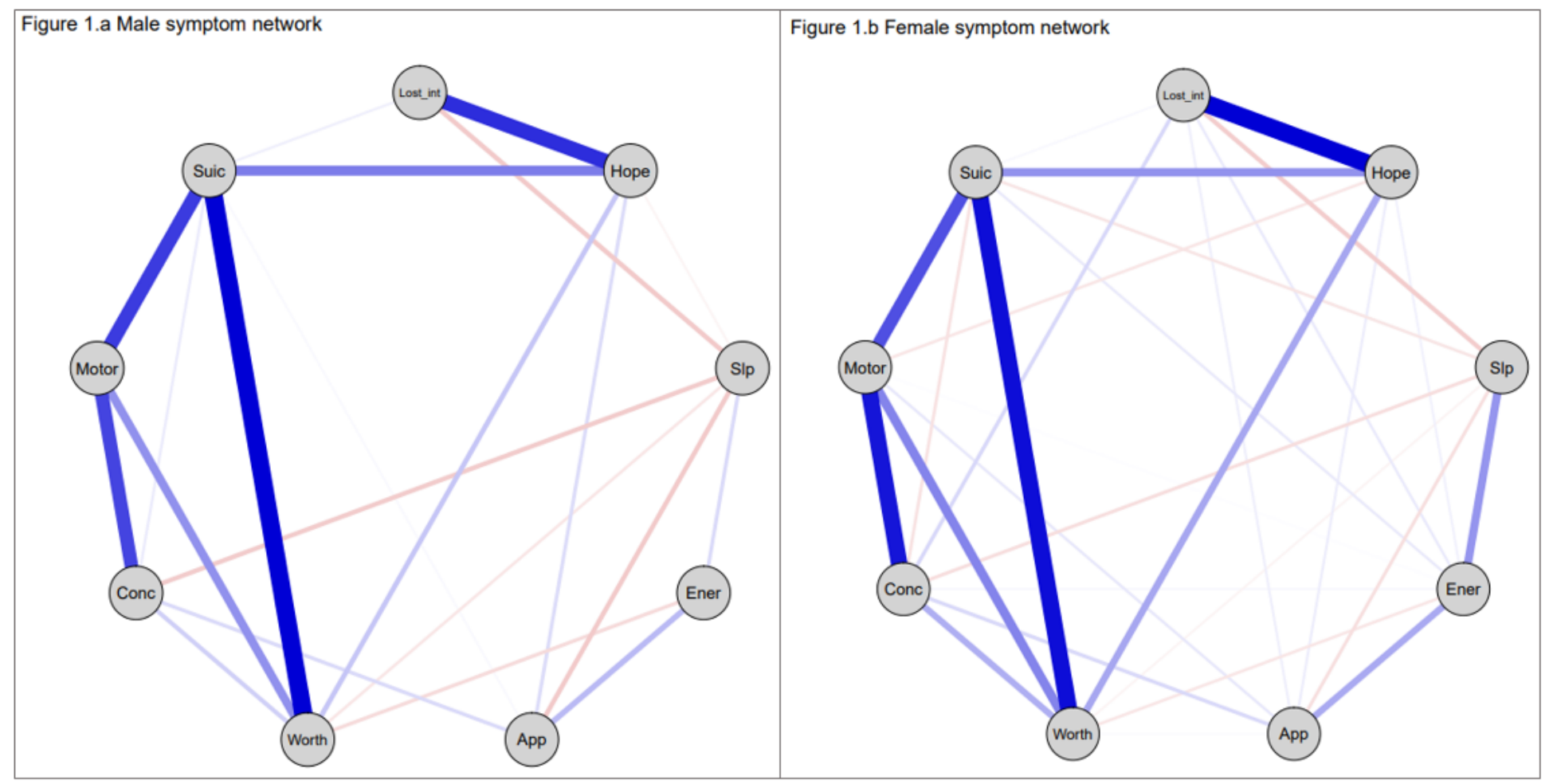

\section{Figure 1}

Graphical LASSO using polychoric correlation Note: In the visualization of the networks, blue lines were used to represent positive edges and red lines were used to represent negative edges. The thicker and more saturated the line, the stronger the connection. Lost_int=loss of interest; Hope=feelings of hopelessness; SIp=sleep difficulty; Ener=low energy or fatigue; App=appetite problems; Worth=feelings of worthlessness; Conc=concentration difficulty; Motor=psychomotor retardation or agitation; Suic=suicide behavior 


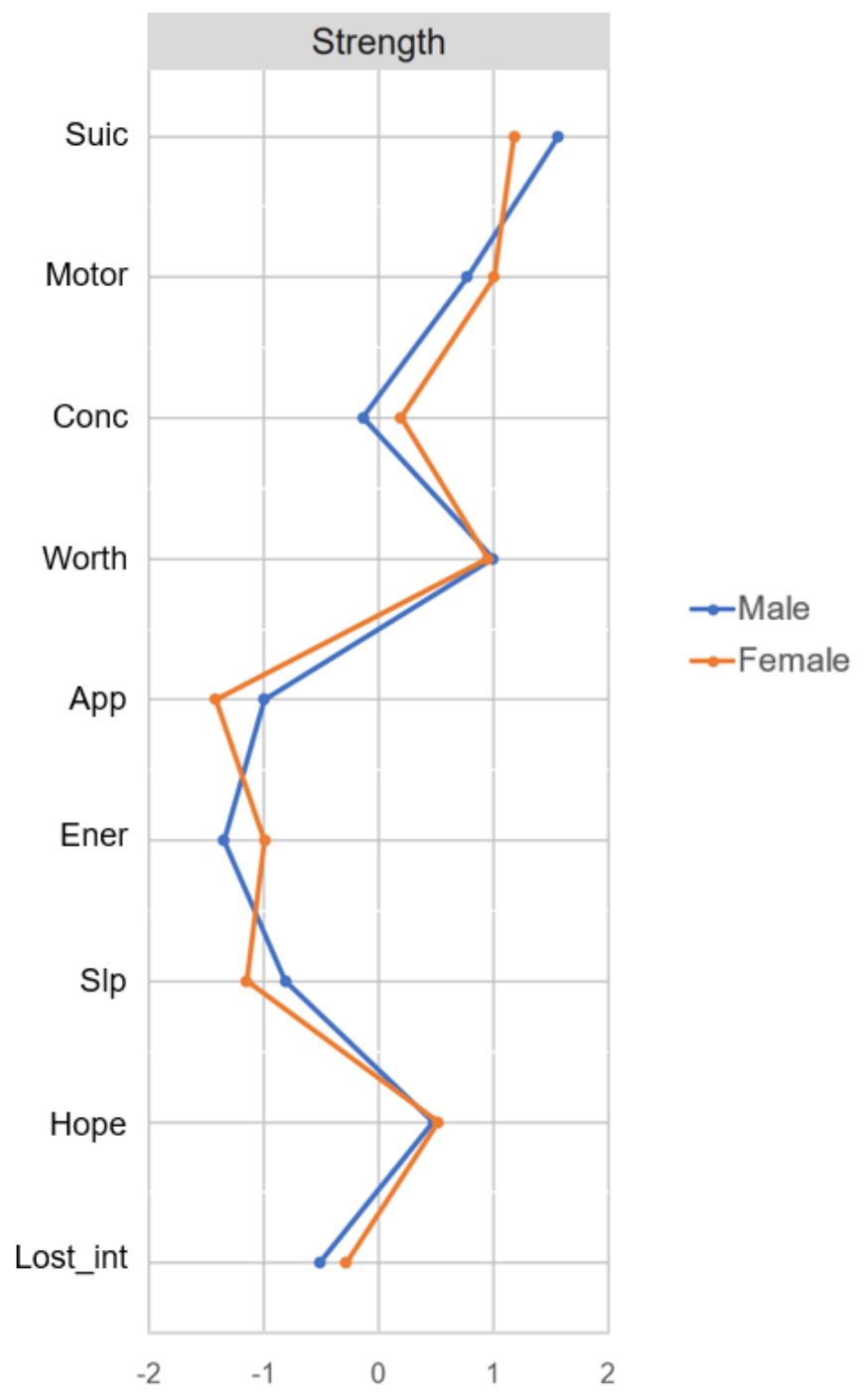

Figure 2

Centrality indices for each PHQ-9 item in the LASSO-regularized partial correlation network Note: Plots depict the normalized (z-scored) values for ease of comparison. The farther right an index is positioned, the higher the node centrality, with the leftmost values representing the least central nodes (see Supplementary Table S1 for more detailed values). Lost_int=loss of interest; Hope=feelings of hopelessness; SIp=sleep difficulty; Ener=low energy or fatigue; App=appetite problems; Worth=feelings of worthlessness; Conc=concentration difficulty; Motor=psychomotor retardation or agitation; Suic=suicide behavior 


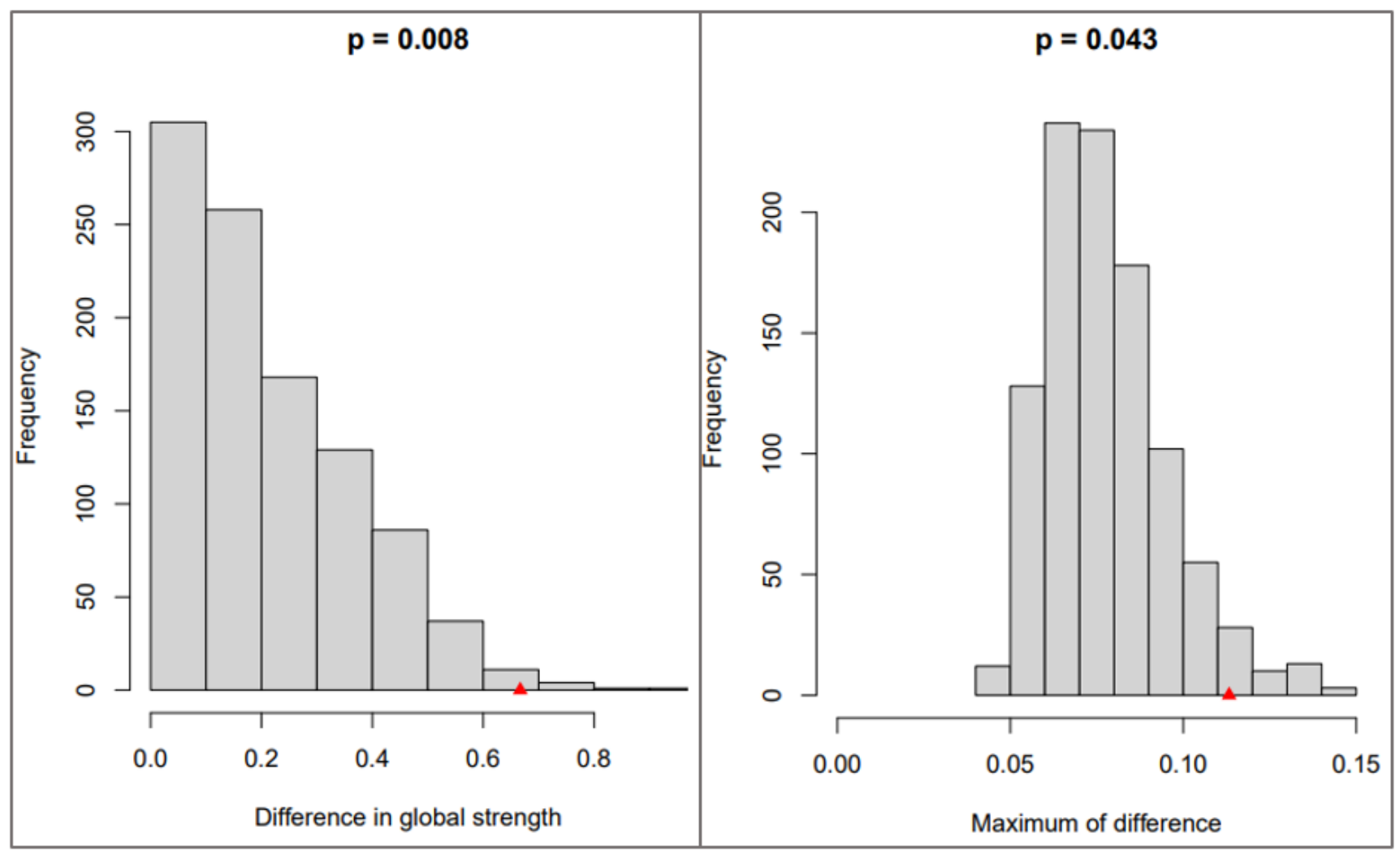

Figure 3

The difference in global strength (left panel) and the maximum difference in edge strength (right panel) Note: The red triangle indicates the test statistic based on the observed data.

\section{Supplementary Files}

This is a list of supplementary files associated with this preprint. Click to download.

- Onlinesupplementaryfiles.docx 